\title{
Gene expression network analysis reveals new transcriptional regulators as novel factors in human ischemic cardiomyopathy
}

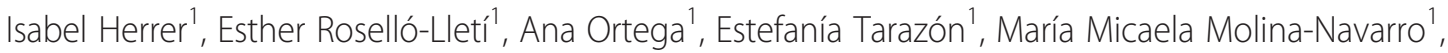
Juan Carlos Triviño ${ }^{2}$, Luis Martínez-Dolz ${ }^{3}$, Luis Almenar ${ }^{3}$, Francisca Lago ${ }^{4}$, Ignacio Sánchez-Lázaro ${ }^{3}$, José Ramón González-Juanatey ${ }^{4}$, Antonio Salvador ${ }^{3}$, Manuel Portolés ${ }^{1}$ and Miguel Rivera ${ }^{*}$

\begin{abstract}
Background: Ischemic cardiomyopathy (ICM) is characterized by transcriptomic changes that alter cellular processes leading to decreased cardiac output. Because the molecular network of ICM is largely unknown, the aim of this study was to characterize the role of new transcriptional regulators in the molecular mechanisms underlying the responses to ischemia.
\end{abstract}

Methods: Myocardial tissue explants from ICM patients and control (CNT) subjects were analyzed by RNA-Sequencing (RNA-Seq) and quantitative Real-Time PCR.

Results: Enrichment analysis of the ICM transcriptomic profile allowed the characterization of novel master regulators. We found that the expression of the transcriptional regulators SP100 (-1.5-fold, p < 0.05), CITED2 (-3.8-fold, p < 0.05), CEBPD (-4.9-fold, $\mathrm{p}<0.05)$ and BCL3 $(-3.3$-fold, $\mathrm{p}<0.05)$ were lower in ICM than in CNT. To gain insights into the molecular network defined by the transcription factors, we identified CEBPD, BCL3, and HIF1A target genes in the RNA-Seq datasets. We further characterized the biological processes of the target genes by gene ontology annotation. Our results suggest that CEBPD-inducible genes with roles in the inhibition of apoptosis are downregulated and that BCL3-repressible genes are involved in the regulation of cellular metabolism in ICM. Moreover, our results suggest that CITED2 downregulation causes increased expression of HIF1A target genes. Functional analysis of HIF1A target genes revealed that hypoxic and stress response genes are activated in ICM. Finally, we found a significant correlation between the mRNA levels of BCL3 and the mRNA levels of both CEBPD $(r=0.73, p<0.001)$ and CITED2 ( $r=0.56$, $p<0.05)$. Interestingly, CITED2 mRNA levels are directly related to ejection fraction $(E F)(r=0.54, p<0.05)$.

Conclusions: Our data indicate that changes in the expression of SP100, CITED2, CEBPD, and BCL3 affect their transcription regulatory networks, which subsequently alter a number of biological processes in ICM patients. The relationship between CITED2 mRNA levels and EF emphasizes the importance of this transcription factor in ICM. Moreover, our findings identify new mechanisms used to interpret gene expression changes in ICM and provide valuable resources for further investigation of the molecular basis of human cardiac ischemic response.

Keywords: Transcriptional regulators, Transcription factor, RNA Sequencing, BCL3, CEBPD, CITED2, SP100, Ischemic cardiomyopathy

\footnotetext{
* Correspondence: miguelrivera492@gmail.com

${ }^{1}$ Cardiocirculatory Unit, Health Research Institute of La Fe University Hospital,

Avd de Fernando Abril Martorell, 106, 46026 Valencia, Spain

Full list of author information is available at the end of the article
} 


\section{Background}

Ischemic cardiomyopathy (ICM) is the most common cause of death in western countries [1]. The factors contributing to ICM are complex. They include microvascular dysfunction, inflammation, disruption of $\mathrm{Ca}^{2+}$ homeostasis, and activation of apoptosis [2,3]. In addition, studies have indicated that a number of fetal and immediate-early genes, including those encoding proteins involved in signal transduction and energy metabolism, are deregulated in the ischemic heart $[4,5]$. These altered processes are associated with a specific gene expression pattern, or transcriptional signature. The transcriptional signature of ICM is thought to arise from ischemic injury or other types of stress stimulus, ultimately resulting in heart failure (HF). Although several global gene expression studies have been carried out in ICM [6], the molecular mechanisms that coordinate the transcriptional profile in ICM are not completely understood.

Gene expression is determined by specific sets of transcription factors (TFs) and by a particular organization, or chromatin structure, of the genome. Several TFs have been implicated in ICM [7]. Specifically, our group identified changes in the protein levels of TFs including nuclear factor of activated T cells 1 (NFAT1), GATA binding protein 4 (GATA4), and nuclear myocyte enhancer factor 2C (MEF2C) in ICM patients [8]. These DNA binding factors are involved in the $\mathrm{Ca}^{2+}$ signaling and may also participate in apoptosis, highlighting the importance of these processes in ICM. Other TFs implicated in ICM include the homeobox protein CSX/ NKX2-5 [9], which participates in the transcriptional regulation of fetal and early-stage genes, the NF-kB pathway activator protein [10], STAT-3 [11], and AP-1 [12]. Despite these findings, the regulatory mechanisms underlying the disruption of essential biological processes in ICM, such as angiogenesis [13] and cellular metabolism, remain to be elucidated.

A traditional approach for identifying the underlying causes of a specific disease is to look for genes that are differentially expressed in disease samples and the appropriate control (CNT) samples. Microarray-based expression profiling has been widely used for this purpose. In addition, gene expression profiling has been used to identify TFs involved in biological processes [14] and in diseases such as dilated cardiomyopathy [15]. There are no genome-wide expression analysis-based studies related to TFs in ICM. RNA sequencing (RNA-Seq) has recently emerged as a precise and sensitive method for mapping and quantifying RNA transcripts [16]. This technology can potentially be used to identify novel transcriptional regulators involved in the molecular mechanisms controlling ICM. Therefore, we aimed to identify novel transcriptional regulators with roles in in ICM development and to characterize their target genes in order to provide novel insights into the mechanisms involved in the responses to ischemia.

\section{Methods}

\section{Ethical approval}

This study was approved by the Biomedical Investigation Ethics Committee of La Fe University Hospital of Valencia, Spain. This work was performed in accordance with the guidelines of the Declaration of Helsinki [17]. Informed written consent was obtained from each patient prior to tissue collection.

\section{Tissue collection}

Left ventricular (LV) tissue samples were collected from human hearts of 13 and 19 patients with ICM undergoing cardiac transplantation and subsequently used in RNA-Seq and quantitative Real-Time PCR (qRT-PCR), respectively. The clinical characteristics of the patients are shown in Table 1. Clinical history, electrocardiography, hemodynamic studies, Doppler echocardiography, and coronary angiography data were available. All patients were functionally classified according to the New York Heart Association (NYHA) criteria and were receiving medical treatment following the guidelines of the European Society of Cardiology [18]. ICM was diagnosed on the basis of the clinical history, Doppler echocardiography, and coronary angiography data.

Table 1 Clinical characteristics of patients with ischemic cardiomyopathy

\begin{tabular}{lll}
\hline & RNA-Seq & qRT-PCR \\
& ICM (n= 13) & ICM ( $=19)$ \\
\hline Age (years) & $54 \pm 7$ & $53 \pm 7$ \\
Gender male (\%) & 100 & 85 \\
NYHA class & $3.5 \pm 0.4$ & $3.1 \pm 0.9$ \\
BMI (kg/m ${ }^{2}$ ) & $26 \pm 4$ & $27 \pm 3$ \\
Hemoglobin (mg/mL) & $14 \pm 3$ & $13 \pm 3$ \\
Hematocrit (\%) & $41 \pm 6$ & $38 \pm 8$ \\
Total cholesterol (mg/dL) & $162 \pm 41$ & $167 \pm 33$ \\
Prior hypertension (\%) & 30 & 31 \\
Prior smoking (\%) & 84 & 58 \\
Prior diabetes mellitus (\%) & 38 & 36 \\
EF (\%) & $24 \pm 4$ & $23 \pm 8$ \\
FS (\%) & $13 \pm 2$ & $12 \pm 4$ \\
LVESD (mm) & $55 \pm 7$ & $53 \pm 7$ \\
LVEDD (mm) & $64 \pm 7$ & $60 \pm 6$ \\
LV mass index (g/cm ${ }^{2}$ ) & $139 \pm 36$ & $130 \pm 34$ \\
\hline
\end{tabular}

Data are showed as the mean value \pm SD or \% of subjects. ICM, ischemic cardiomyopathy; NYHA, New York Heart Association; BMI, body mass index; $E F$, ejection fraction; $F S$, fractional shortening; LVESD, left ventricular end-systolic diameter; LVEDD, left ventricular end-diastolic diameter; LV mass index, left ventricular mass index. 
CNT LV samples were obtained from the hearts of six (RNA-Seq) or seven (qRT-PCR) healthy donors whose hearts could not be transplanted due to surgical reasons or blood type incompatibility. The cause of death in these individuals was cerebrovascular or motor vehicle accident. All donors had normal LV function and had no history of myocardial disease or active infection at the time of transplantation.

Fresh transmural samples were recovered from near the apex of the left ventricle at the time of transplantation. Tissue samples were maintained in $0.9 \% \mathrm{NaCl}$ at $4^{\circ} \mathrm{C}$ for a maximum of 6 hours from the time of coronary circulation loss and then frozen at $-80^{\circ} \mathrm{C}$ until RNA extraction.

\section{RNA extraction}

Heart samples were homogenized with TRIzol $^{\circ}$ Reagent in a TissueLyser LT (Qiagen; UK). All RNA extractions were performed using a PureLink $^{\circ}$ RNA Mini Kit (AmbionLife Technologies; CA, USA), according to the manufacturer's instructions. RNA was quantified using a NanoDrop1000 spectrophotometer (Thermo Fisher Scientific; UK) and the purity and integrity of the RNA samples were measured using an Agilent 2100 Bioanalyzer with the RNA 6000 NanoLabChip kit (Agilent Technologies; Spain). All samples displayed a 260/280 nm absorbance ratio greater than 2.0 and RNA integrity numbers $\geq 9$.

\section{RNA-Seq}

PolyA-RNA was isolated from $25 \mu \mathrm{g}$ of total RNA using the MicroPoly(A) Purist kit (Ambion, USA). Total PolyA-RNA was used to generate whole transcriptome libraries for sequencing on the SOLiD 5500XL platform following the manufacturer's recommendations (Life Technologies; CA, USA). Amplified cDNA quality was analyzed using the Bioanalyzer 2100 DNA 1000 kit (Agilent Technologies; Spain) and quantified using the Qubit 2.0 Fluorometer (Invitrogen; UK). Whole transcriptome libraries were used to make SOLiD templated beads following the SOLiD templated bead preparation guide. This protocol consisted of an RNA enrichment and chemical modification step, followed by a clonal amplification step. Bead quality was estimated based on work flow analysis parameters. The samples were sequenced using the 50625 paired-end protocol, generating $115 \mathrm{nt}$ sequences consisting of $75 \mathrm{nt}$ plus $35 \mathrm{nt}$ (Paired-End) + 5 nt (Barcode). Quality data was measured using software parameters of the SOLiD Experimental System.

\section{Computational analysis of RNA-Seq data}

The initial whole transcriptome paired-end reads obtained from sequencing were mapped against the latest version of the human genome (version GRchr37/hg19) using the Life Technologies mapping algorithm (http:// www.lifetechnologies.com/), version 1.3. For both forward and reverse reads, the seed was the first $25 \mathrm{nu}$ cleotides with a maximum of 2 mismatches allowed. Additional file 1: Table S1 describes the main statistical parameters of the mapping analysis. The aligned records were reported in BAM/SAM format [19]. Insufficient quality reads (phred score $<10$ ) were eliminated using Picard Tools software, version 1.83 (http://picard.sourceforge.net/). Gene prediction was estimated using Ensembl ID and the Cufflinks method for de novo assembly [20]. After alignment the read counts or gene expression levels were calculated using HTSeq software, version 0.5.4p3 (http://www-huber.embl.de/users/anders/ HTSeq/). Differential expression analysis between conditions was performed using the edgeR method, version 3.2.4 [21]. This method uses a Poisson distribution to model genic read counts following normalization based on size factors and variance; therefore, this software allows normalization of RNA-Seq data based on sequencing depth, GC content, and gene length for analysis of differential expression. We selected differentially expressed genes with a $p$-value $<0.05$ and a fold change of at least 1.5. Primary RNA-Seq data were submitted to the public database Gene Expression Omnibus (GEO) repository, and the accession number to the data file is GSE55296 (http://www.ncbi.nlm.nih.gov/geo/query/acc.cgi?acc=GS E55296).

Finally, we assessed the technical variation by Pearson correlation analysis using the R-statistical software, version 3.0.3 (http://www.r-project.org).

\section{Principal component analysis}

To classify heart samples, a principal component analysis (PCA) was performed with the RNA-Seq data. A scatter plot was produced in order to visualize the differences between the sample sets based on each sample's gene expression profile. This analysis was performed using R-statistical software, version 3.0.3 (http://www.rproject.org).

\section{Gene set enrichment analysis}

To identify over-represented TFs from RNA-Seq data, we used a web-based interactive application called ChIP Enrichment Analysis (ChEA) [22]. The ChEA database contains data from genome-wide ChIP studies and therefore takes into consideration the chromatin state of the cell. TFs showing a p-value $<0.05$ were considered significant.

\section{qRT-PCR}

One microgram of RNA was reverse-transcribed to cDNA using the M-MLV enzyme (Invitrogen, UK). qRT-PCR 
was performed in duplicate using the TaqMan protocol in a ViiA7 Fast Real-Time PCR System according to the manufacturer's instructions (Applied Biosystems; USA). The following TaqMan probes were designed and obtained from Applied Biosystems: SP100 nuclear antigen (SP100) (Hs00162109_m1), Cbp/p300-interacting transactivator 2 (CITED2) (Hs00366696_m1), CCAAT/enhancer binding protein delta (CEBPD) (Hs00270931_s1) and Bcell CLL/lymphoma 3 (BCL3) (Hs00180403_m1). The housekeeping genes GAPDH (Hs99999905_m1), PGK1 (Hs99999906_m1), and TFRC (Hs00951083_m1) were used as reference genes. $\Delta \Delta \mathrm{Ct}$-based fold change calculations were used to determine relative transcript quantity [23]. Individual fold changes were calculated comparing each ICM $\Delta \mathrm{Ct}$ value with the corresponding $\mathrm{CNT}$ pooling $\Delta \mathrm{Ct}$ values.

\section{TF target genes prediction}

To decipher the transcriptional regulatory networks, we identified the TF target genes that were differentially expressed between ICM patients and CNT individuals. TF target gene prediction was carried out using the transcriptional regulatory element database (TRED) (http:// rulai.cshl.edu/TRED), TFactS (http://www.tfacts.org), and the ChIP-X database, a component of the ChEA software. All three informatics tools encompass the prediction of TF regulation based on TF binding motifs and experimental evidence.

\section{TF target genes functional annotation}

Functional annotation analysis of differentially expressed genes was performed using the Database for Annotation, Visualization and Integrated Discovery (DAVID, version 6.7). Gene ontology (GO) terms that had a $p$-value $<0.05$ were selected [24].

\section{Statistics}

Data are expressed as the mean \pm standard deviation (SD). The Kolmogorov-Smirnov test was applied to evaluate the data distribution. Clinical characteristics were compared using Student's $t$-test for continuous variables and Fisher's exact test for discrete variables. Significant mean differences in mRNA levels between groups were determined using Student's $t$-test. Pearson's correlation coefficient was calculated to analyze the association between mRNA levels and the relationship between mRNA expression levels and clinical parameters. A $p$-value $<0.05$ was considered significant. Statistical analysis was performed using the Statistical Package for Social Sciences, version 20.0 (IBM SPSS Inc., Chicago. IL, USA) and GraphPad Prism software version 6.0 (http://www.graphpad.com).

\section{Results}

\section{Clinical characteristics of patients}

We analyzed by RNA-Seq a total of 19 LV tissue samples corresponding to hearts from 13 ICM patients undergoing cardiac transplantation and six non-diseased CNT donors. ICM patients were $100 \%$ male, and were a mean age of $54 \pm 7$ years. All of them had a NYHA functional classification of III-IV and previously had been diagnosed with significant comorbidities, including hypertension and hypercholesterolemia (Table 1). The CNT group comprised $83 \%$ men and the mean age was $53 \pm$ 10 years.

We used a greater sample size for qRT-PCR validation of up to $26 \mathrm{LV}$ samples corresponding to hearts from 19 ICM patients and seven non-diseased CNT donors. ICM patients were $85 \%$ male, and were a mean age of $53 \pm$ 7 years. Their clinical characteristics are shown as the mean value \pm SD in Table 1 . No significant differences were found in clinical parameters between the two ICM groups. The CNT group comprised $75 \%$ men and the mean age was $51 \pm 9$ years.

\section{RNA-Seq results}

To investigate the transcriptomic changes accompanying ICM, we performed a large-scale expression screen using RNA-Seq technology. Nineteen heart samples were used for the analysis (ICM, $n=13$ and CNT, $n=6$ ). Primary RNA-Seq data were submitted to the public database Gene Expression Omnibus (GEO) repository [accession number GSE-55296]. Results of pairwise Pearson-correlations between samples showed no significant differences (Additional file 2: Figure S1). We applied a threshold of 0.85 . Since inclusion of transcripts expressed at very low levels increases the risk of false discovery, a minimum of five normalized transcript read counts was used as the cut-off point. Transcripts from 13,374 genes were identified in CNT hearts and from 13,464 genes were detected in ICM tissue by RNA-Seq. For transcript identification we used Ensembl ID information and the Cufflinks method for the unknown genes. Expressed genes were divided into groups according to their relative expression levels (Figure 1A-B). We found that 943 and 1,026 genes showed the lowest levels of expression, represented by five and ten read counts in CNT and ICM, respectively. While the majority of genes in CNT $(88 \% ; 11,748 / 13,374)$ and in $\operatorname{ICM}(87 \% ; 11,780 / 13,464)$ displayed between 10 and 1000 read counts, only 13 genes in CNT hearts and in ICM samples were highly expressed, with $\geq 100,000$ read counts (Figure 1A-B). The most abundantly transcribed genes were MT-ND4 (NADH dehydrogenase subunit 4, a mitochondrial-encoded gene), with a read count of 2,422,108, in CNT and MT-COI (cytochrome c oxidase subunit I, a mitochondrial-encoded gene) in ICM, with a read count of 2,170,339. Most prevalent among the genes 


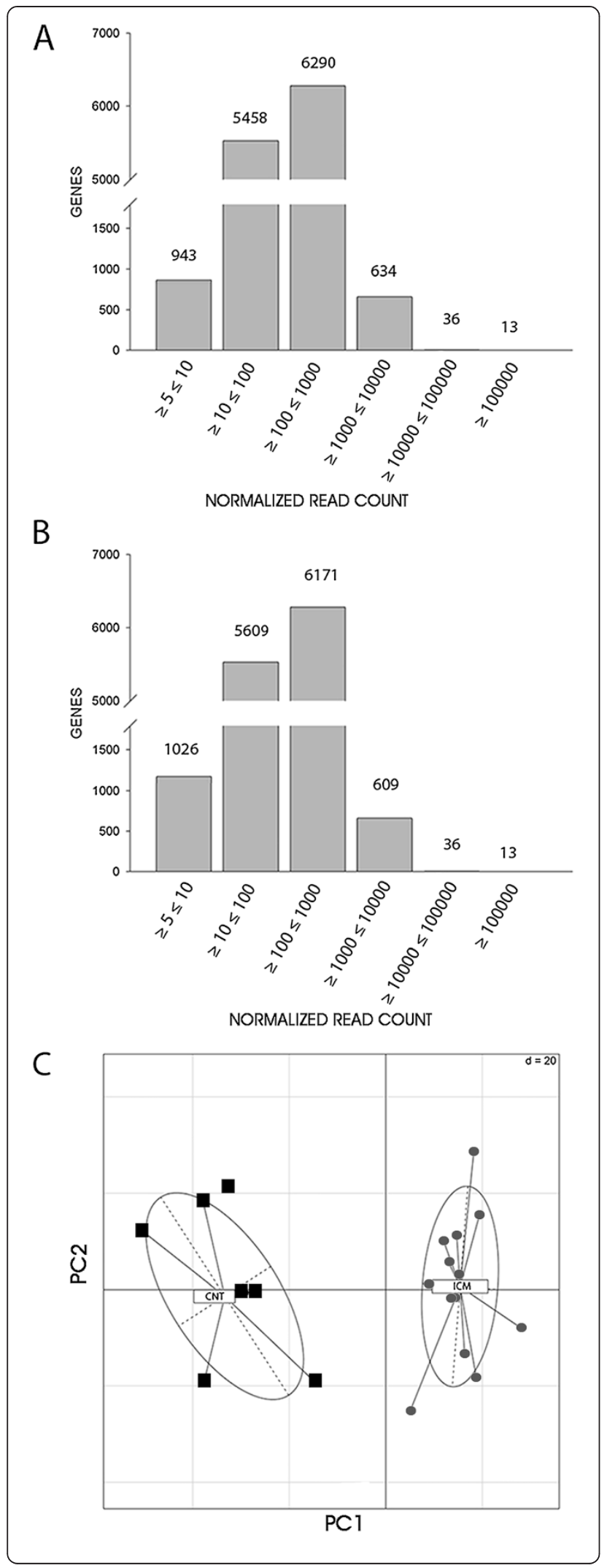

Figure 1 Transcriptomic profiling of heart samples by RNA-Seq. The number of genes and their relative expression levels represented by transcript normalized read counts in controls (A) and ICM patients (B). (C) Principal Component Analysis of heart samples were cluster on the basis of their gene expression profile. Samples are represented by points at CNT for controls and ICM for patients. Proportions of variances showed that $0.95 \%$ of the differences among the sample groups could be explained by PCA component 1 (PC1) and $0.233 \%$ by PCA component 2 (PC2).

with read counts of approximately 100,000 in both types of samples were those encoding cytoskeletal components, including MYL2, ACTLC1, or DES.

Significance analysis of the RNA-Seq results revealed a total of 1,334 genes that were differentially expressed in ICM patients vs CNT; 649 genes were upregulated $(\geq 1.5$ fold, $\mathrm{p}<0.05)$ and 685 genes were downregulated $(\leq 1.5$ fold, $\mathrm{p}<0.05)$. These genes encompass the ICM transcriptome signature. In order to validate these genetic differences, clustering analysis of the samples was performed by PCA with the differentially genes. When PCA is applied to data, samples with similar trends in their gene expression profiles tend to cluster close together in the plot. PCA demonstrated that the gene expression profiles showed a clear distinction between ICM patients and CNT group (Figure 1C).

\section{TF enrichment analysis}

In order to identify key TFs mediating the differential expression of genes in ICM, the transcriptional signature was analyzed in ChEA database containing $94 \%$ of these genes. Using ChEA enrichment analysis, we identified TFs associated with ICM at a $p$-value $<0.05$, calculated by Fisher's exact test (Additional file 3: Table S2). Figure 2 shows relevant TFs with a $p$-value $<0.01$. These TFs include those previously associated with ICM, including GATA4 (1.7-fold enrichment), NKX2.5 (1.7-fold enrichment), STAT3 (2.4-fold enrichment), and EP300 (2-fold enrichment) $[8,9,11,25]$, demonstrating the reliability of this database. In addition, TFs such as ESR1 (2.2-fold enrichment), and the pluripotency markers SOX2 (2.9-fold enrichment) and NANOG (2-fold enrichment), have not been previously implicated in human ICM. HIF1A (3.02-fold enrichment) was significantly over-represented in ICM, as were CEBPD (3-fold enrichment), and BCL3 (1.7-fold enrichment) (Figure 2). Furthermore, RNA-Seq data showed that gene expression levels of $C E B P D, B C L 3$, and the HIF1A repressor, CITED2, significantly decreased in ICM patients (Table 2).

Not all TFs identified in the enrichment analysis were differentially expressed according our RNA-Seq results. Since protein levels, post-transcriptional modifications, and other factors that affect TF regulatory function can also influence the activities of target genes, it is not 


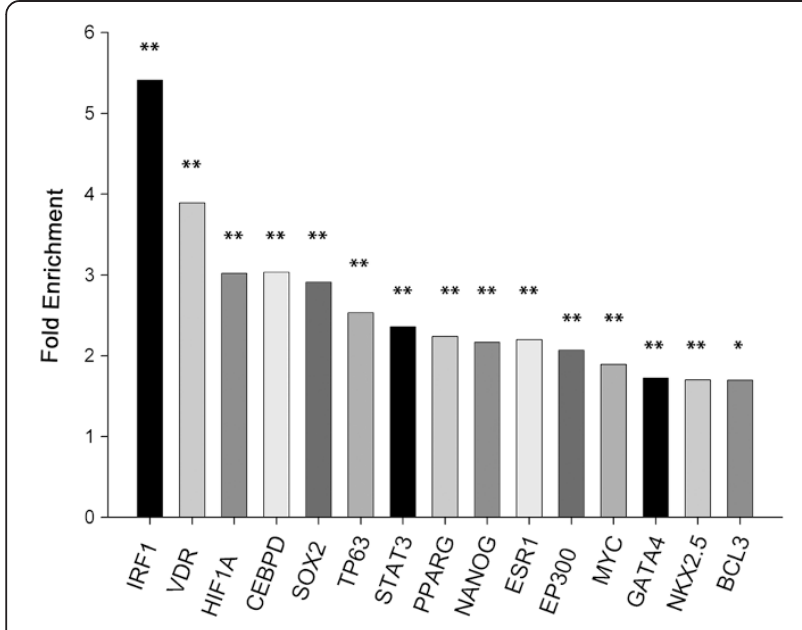

Figure 2 Transcription factor (TF) enrichment analysis. Relevant TFs identified using the ChEA database and based on the RNA-Seq genetic profile. The graph shows the official name and the fold enrichment of each TF in ICM vs CNT. Fold enrichment was calculated by an algorithm considering target genes of our list/target genes ChIP-X database relation. All comparisons were statistically significant $\left({ }^{*} p<0.01,{ }^{* *} p<0.001\right)$.

surprising to find overrepresentation of a specific TF signaling pathway without a change in the gene expression of the TF.

On the other hand, the TFs NFAT1 and MEF2C, already found in ICM patients in previously studies [8], are not included in the ChIP-X database. Because ChIP studies that profile the binding of NFAT1 and MEF2C are not available, these proteins were not identified in the TF enrichment analysis.

\section{qRT-PCR analysis}

Next, we used qRT-PCR to validate the RNA-seq data indicating differences between ICM and CNT samples in the mRNA levels of SP100, CITED2, CEBPD, and BCL3, four transcriptional regulators that have not been implicated previously in ICM. SP100 encodes a transcriptional coactivator/corepressor that regulates DNA-binding by other TFs, and CITED2, CEBPD and BCL3 encode TF that are closely involved in stress or hypoxia responses [26]. Given the importance of these processes in disease development, we considered it interesting to further study their

Table 2 Selected genes analyzed by RNA-Seq and used for qRT-PCR validation

\begin{tabular}{llll}
\hline Gene ID & Gene symbol & $\begin{array}{l}\text { Fold change } \\
\text { (ICM vs CNT) }\end{array}$ & p value \\
\hline ENSG00000067066 & SP100 & -1.8994 & 0.0005 \\
ENSG00000164442 & CITED2 & -1.5631 & 0.0215 \\
ENSG00000221869 & CEBPD & -3.2139 & $2.20 \mathrm{E}-09$ \\
ENSG00000069399 & BCL3 & -1.9970 & $1.38 \mathrm{E}-05$ \\
\hline
\end{tabular}

regulatory mechanisms. As a result, we validated the expression levels of these genes.

qRT-PCR confirmed lower expression of SP100 (-1.5fold, $\mathrm{p}<0.05$ ) in ICM than in CNT (Figure 3). In addition, the expression of the TF genes CITED2 (-3.8-fold, $\mathrm{p}<$ 0.05), CEBPD (-4.9-fold, $\mathrm{p}<0.05$ ) and BCL3 (-3.3-fold, $\mathrm{p}<0.05)$ were significantly lower in ICM $v s$ CNT hearts (Figure 3). Further verification of fold change values for these genes was performed by comparing qRT-PCR and RNA-Seq data from the same 10 patient samples. The direction and degree of fold changes were similar in all cases for RNA-Seq and qRT-PCR. For most of the genes analyzed there was a good correlation between both techniques, although, generally, greater fold change differences were detected for the downregulated genes by qRT-PCR (Figure 4).

\section{TF target genes analysis}

To gain insights into alterations in the gene regulatory network in ICM patients, we identified downstream responsive genes of CEBPD, BCL3, and HIF1A and then compared RNA-Seq expression levels of these genes between ICM and CNT LV samples. We performed an integrative bioinformatic approach using three prediction databases: TRED, TFactS, and ChEA. The results from the database analyses were complemented with results from a previous study by Yang et al. [27], which determined the transcriptomic signature of cardiomyocytes overexpressing BCL3. Although the protein encoded by SP100 binds to chromatin and regulates gene expression [28], is not defined as a TF so we did not use it in the following analysis.

Results from the target predictions showed that a total of 25 CEBPD target genes were downregulated in the cardiac tissue of ICM patients, and that these targets encompassed members of the TGF $\beta$ family, including TGFBR2 and BMPR1B. In addition, 70 BCL3 target genes were downregulated in ICM patients, including genes encoding TFs (STAT2, FOSL2, GABPR2), cytokines (CXCL1, CXCL10), and growth factor receptors (TGFBR3). CITED2 is a TF without typical DNA binding domains, and a well-known corepressor of HIF1A transcriptional activation [29]; so we further studied the potential function of HIF1A and CITED2 through changes in the expression of HIF1A target genes. We found that 17 HIF1A target genes were upregulated in ICM patients, including well-known hypoxic genes such as EGLN3 and IGFBP3 [30]. All target genes of CEBPD, BCL3, and HIF1A are shown in Additional file 4: Table S3.

We next analyzed the functional downstream effects of the differentially expressed genes using the DAVID software for $\mathrm{GO}$ annotation. A significant enrichment $(p<0.05)$ indicates specific biological processes that are affected when the corresponding genes are altered. GO 


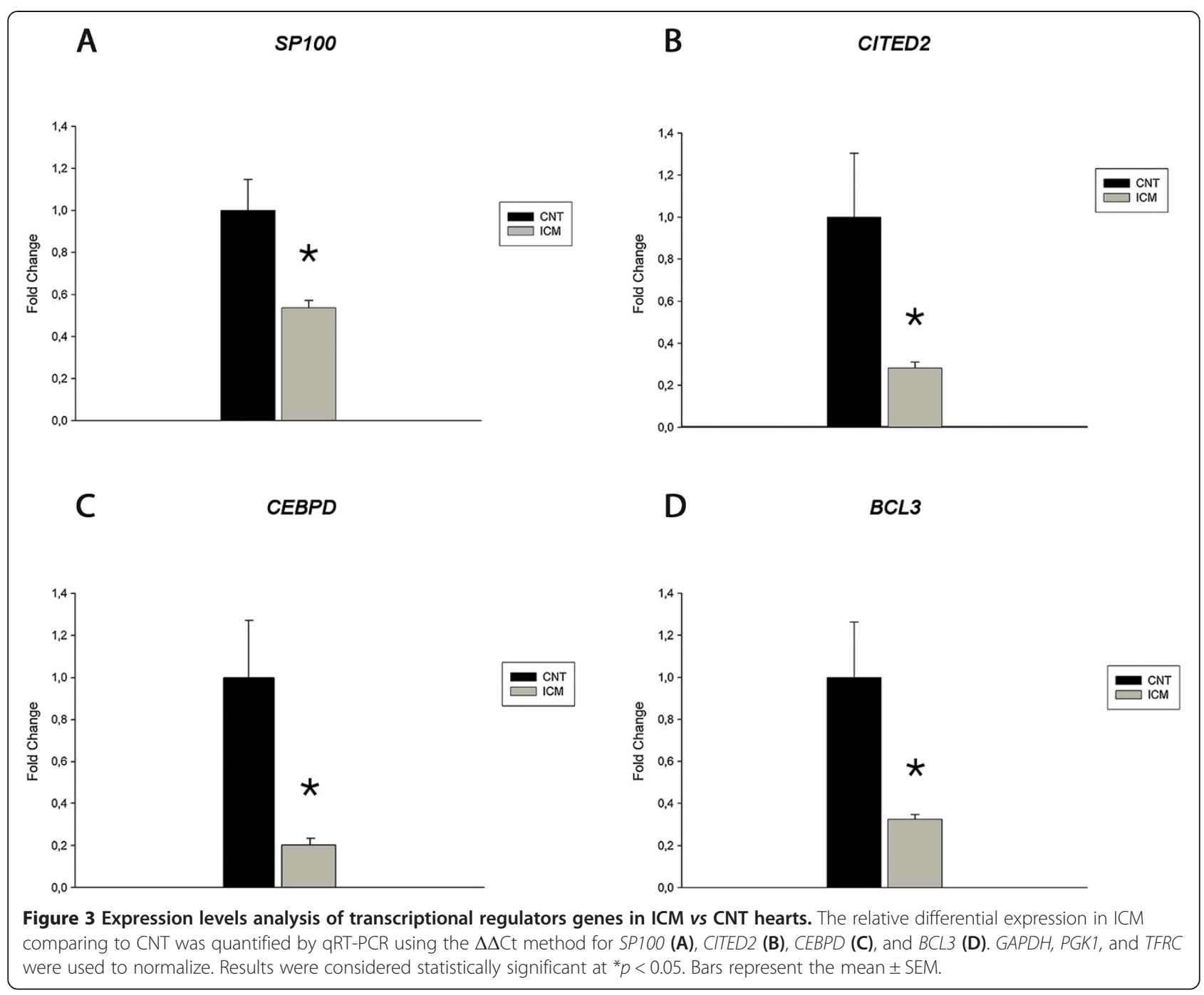

results indicated that the downregulated CEBPD target genes are mainly involved in activating apoptosis and that the BCL3 target genes are involved in energy metabolism, including the regulation of responses to glucose levels (Table 3). Finally, genes regulated by HIF1A participate in hypoxic and stress responses (Table 3). Additional GO terms related to biological processes and significantly represented by genes downstream of CEBPD, BCL3 and HIF1A are shown in Additional file 5: Table S4.

\section{Relationship between gene expression levels and LV function parameters}

In order to determine a potential association between the dysregulated genes in ICM, we determined whether there was some relation between changes in gene expression levels. The results showed that BCL3 mRNA levels were significantly correlated with $C E B P D(\mathrm{r}=0.73$, $\mathrm{p}<0.001)$ and CITED2 $(\mathrm{r}=0.56, \mathrm{p}<0.05)$ mRNA levels (Figure 5A-B). We also investigated the association between the mRNA expression levels and the clinical parameters of the patients. Ejection fraction (EF) represents the fraction of blood that leaves the left and right ventricles when the heart contracts. The EF clinical data from the ICM patients were available for 14 of the samples used in qRT-PCR analysis. We found a direct correlation between CITED2 mRNA expression levels and LV EF $(r=0.54, \mathrm{p}<0.05)$ (Figure 5C).

\section{Discussion}

The regulation of gene expression is dependent on the expression profiles of specific TFs, and also involves chromatin remodeling complexes that regulate the access of TF to DNA sequences. These mechanisms represent a key node in maintaining cell homeostasis, and the dysregulation of these interactions has been associated with diseases, including ICM [31]. While TFs involved in altered $\mathrm{Ca}^{2+}$ homeostasis, inflammation, and apoptosis disruption have been associated with ICM [2], little is known about the specific signaling response that is affected. The role of TFs in orchestrating other important 


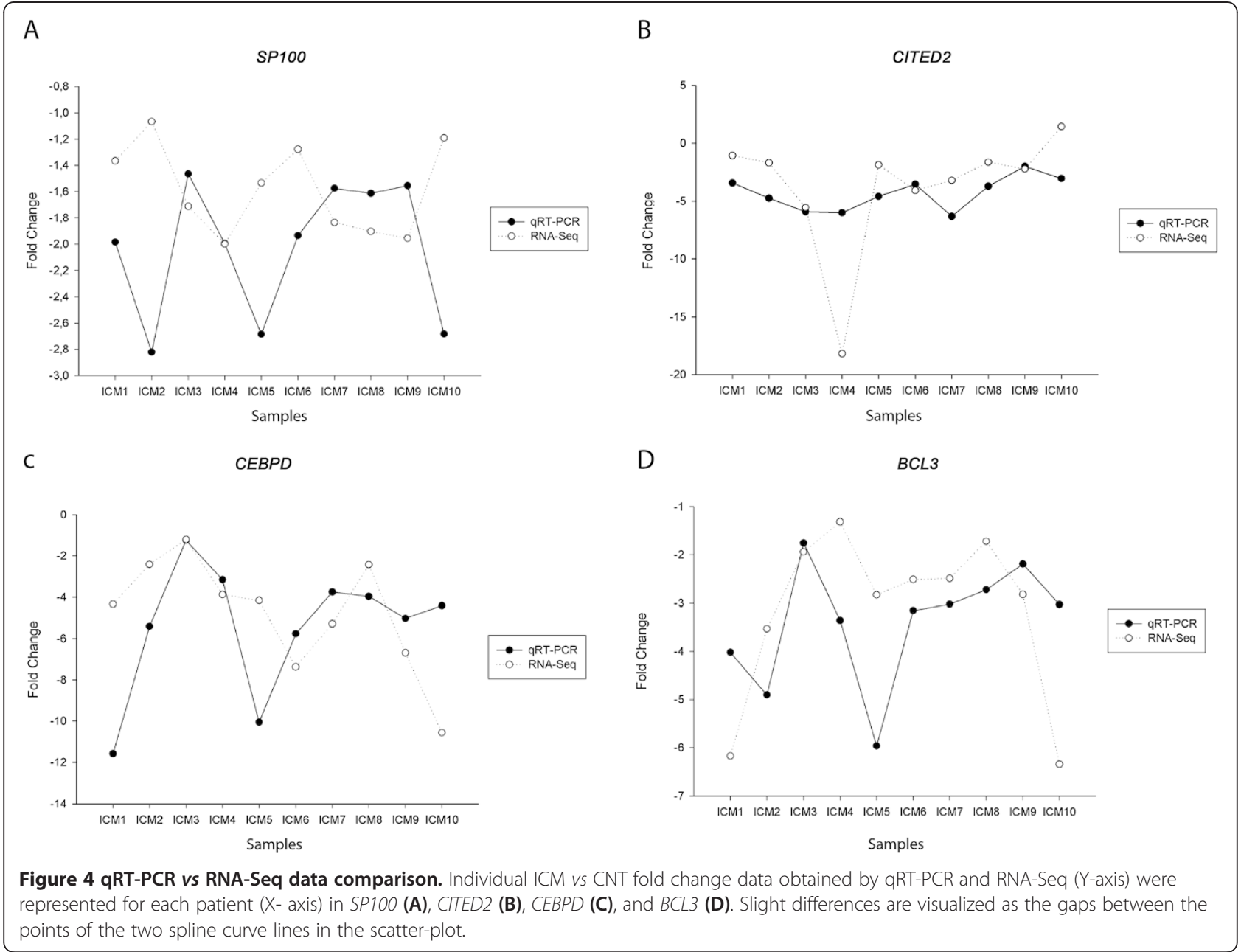

processes, such as stress and hypoxic responses, is also not clearly understood. Moreover, although previous microarray studies have identified ICM specific gene expression profiles [6,32], the regulatory mechanism underlying these profiles has not yet been determined. We hypothesized that novel regulatory factors could function to coordinate the gene expression signatures involved in the etiology or development of ICM.

In this work, we have described the spectrum of genes expressed in CNT and ICM heart tissue, identified by RNA-Seq. Transcriptomic analysis indicated that genes that encode components of the respiratory electron transport are those that are among most abundantly expressed. The analysis also indicated that cytoskeleton genes are highly expressed, demonstrating the significant abundance of their components in the heart. The differential gene expression profile between ICM and CNT LV samples was used to perform an enrichment analysis to identify relevant TFs. Enrichment analysis of RNASeq results identified TFs previously found to be involved in ICM, including EP300, GATA4 [8], STAT-3
[11] and NKX2-5 [33]. Moreover, the identification of ESR1 as a regulatory factor in human ICM in this study correlates with the association of specific polymorphisms in the ESR1 gene to coronary heart disease [34]. In addition, evidence of the pluripotency of the TFs MYC, NANOG, and SOX2 in ICM suggests that they may participate in the fetal gene re-expression program in ICM [35].

We next focused our study on analyzing the expression levels of the TFs genes CITED2, CEBPD, and BCL3, which are involved in the regulation of the stress or hypoxic response, and on the SP100 gene, which encodes a heterochromatin binding factor. qRT-PCR analysis validated the downregulation of these transcriptional regulators in ICM.

The SP100 gene encodes a nuclear body component that has an important role in chromatin-mediated gene regulation [36]. It has been proposed that suppression of SP100 activates cell immortalization, leading to genomic instability and cytoplasmic sequestration of p53 [37]. Interestingly, ICM hearts are characterized by presenting DNA fragmentation [38], and recently it has been shown 
Table 3 Identification and functional annotation analysis of TF target genes

\begin{tabular}{|c|c|c|c|}
\hline FT & GO & p-value & Genes \\
\hline$\overline{C E B P D}$ & System development & $5.00 \mathrm{E}-05$ & $\begin{array}{l}\text { CITED2, RGMA, BMPR1B, CALCRL, FGF7, IL1B, IRX3, LRRC4C, } \\
\text { RXFP2, TGFBR2, TNFRSF11A }\end{array}$ \\
\hline CEBPD & Negative regulation of apoptosis & $1.10 \mathrm{E}-02$ & CITED2, RIPK2, IL1B, RXFP2 \\
\hline CEBPD & Negative regulation of programmed cell death & $1.20 \mathrm{E}-02$ & CITED2, RIPK2, IL1B, RXFP2 \\
\hline CEBPD & Blood vessel morphogenesis & $3.20 \mathrm{E}-02$ & $C T H, I L 1 B, R X F P 2, T G F B R 2$ \\
\hline CEBPD & Negative regulation of cell proliferation & $1.20 \mathrm{E}-02$ & CITED2, TGFBR2, IL1B \\
\hline $\mathrm{BCL3}$ & Regulation of metabolic process & $4.80 \mathrm{E}-04$ & $\begin{array}{l}\text { PFKFB2, ABCA1, ATPIF1, FOSL2, GABPB2, SP110, TSPYL2, } \\
\text { ATF7, A2M, ANKRD1, CALCRL, GDF7, HR, MLYCD, MYD } 88, \\
\text { NRAA1, NR4A3, PER1, PIM1, PDCD4, PSMBS, STAT2, SOAT1, } \\
\text { TXNIP, TGFBR2, TGFBR3, UBB, UBE2L6, ETS2 }\end{array}$ \\
\hline $\mathrm{BCL} 3$ & Regulation of nitrogen compound metabolic process & $6.30 \mathrm{E}-03$ & $\begin{array}{l}\text { ABCA1, ATPIF1, FOSL2, GABPB2, SP110, TSPYL2, ATF7, ANKRD1, } \\
\text { CALCRL, GDF7, HR, MYD } 88, \text { NR4A1, NR4A3, PER1, PIM1, } \\
\text { PDCD4, STAT2, TXNIP, TGFBR3, UBB, ETS2 }\end{array}$ \\
\hline $\mathrm{BCL} 3$ & Response to glucose stimulus & $1.60 \mathrm{E}-02$ & PFKFB2, TXNIP, TGFBR2 \\
\hline $\mathrm{BLC} 3$ & Response to hexose stimulus & $1.80 \mathrm{E}-02$ & PFKFB2, TXNIP, TGFBR2 \\
\hline $\mathrm{BCL} 3$ & Response to monosaccharide stimulus & $1.80 \mathrm{E}-02$ & PFKFB2, TXNIP, TGFBR2 \\
\hline CITED2/HIF1A & Response to hypoxia & $9.80 \mathrm{E}-06$ & ANGPTL4, EGLN3, NOL3, KCNA5 \\
\hline CITED2/HIF1A & Response to oxygen levels & $1.20 \mathrm{E}-05$ & ANGPTL4, EGLN3, NOL3, KCNA5 \\
\hline CITED2/HIF1A & Response to stress & $9.20 \mathrm{E}-04$ & ANGPTL4, CCND1, EGLN3, MIF, NOL3, KCNA5, TFF3 \\
\hline CITED2/HIF1A & Regulation of cell proliferation & 8.00E-03 & CCND1, DPT, EGLN3, ADM, IGFBP3 \\
\hline
\end{tabular}

that cytosolic sequestration of p53 facilitates mitochondrial dysfunction and HF in mice [39]. These data suggest that decreased SP100 expression in ICM may be associated with these molecular heart alterations.

To gain insights into the molecular network of the differentially expressed TFs, we studied their potential roles in ICM by characterizing changes in the expression of their putative target genes and alterations in associated processes. Bioinformatics analysis of the RNA-Seq data identified target genes of CEBPD, BCL3, and CITED2 that are potentially involved in ICM.

Twenty-five CEBPD target genes were downregulated in ICM patients, some of which are implicated in apoptosis via downregulation of RIPK2 and RXFP2 [40]. Many of the 70 BCL3 target genes that are downregulated in ICM patients are involved in the regulation of energetic biological processes and in the inhibition of the glucose response [41]. This suggests that BCL3 downregulation may be the underlying cause of the metabolic disruption of the carbohydrate responses in these patients. These data are consistent with a previous study suggesting cooperation between BCL3 and PCG$1 \alpha$ in the coordination of inflammation and energy metabolism in the heart [31]. It should be noted, however, that our RNA-Seq data did not detect significant differences in PCG-1 $\alpha$ expression between ICM and CNT samples. So the physiological mechanism may not be completely disrupted by the altered BCL3 response in the disease. BCL3 is an $\mathrm{IkB}$ protein that associates tightly with p50 or p52 homodimers as an activator component of the NF-kB pathway $[42,43]$, and it has been shown that NF-kB activation is characteristic of failing myocardium $[44,45]$. Functional characterization of genes downstream of $B C L 3$ downstream genes did not identify the NF-kB pathway. Activation of the NF-kB pathway in ICM patients may be mediated by mechanisms that are independent of BCL3.

Given the well-known role of CITED2 as a corepressor of HIF1A transcriptional activation [46], we evaluated the changes in expression of HIF1A target genes in ICM and CNT tissues. We found that 17 HIF1A target genes are upregulated in ICM. The hypoxic and stress response genes were highly represented. Previous studies have suggested that multiple pathways act cooperatively to fine-tune transcriptional responses [47]; however, our data are the first to show cooperative transcriptional regulation of the stress response and hypoxic response pathways by HIF1A in ICM (Figure 6).

Hypoxia plays an essential role in cellular and systemic homeostasis. Hypoxia-regulated genes control many cellular processes, including the switch from oxidative to glycolytic metabolism, stimulation of oxygen release, and angiogenesis, and these genes even are involved in cardioprotection $[48,49]$. Lei et al. [50], for example, suggested that chronic activation of the HIF pathway in ischemic hearts is maladaptive and contributes to cardiac degeneration and progression to heart failure. The downregulation of CITED2 also may contribute to the 


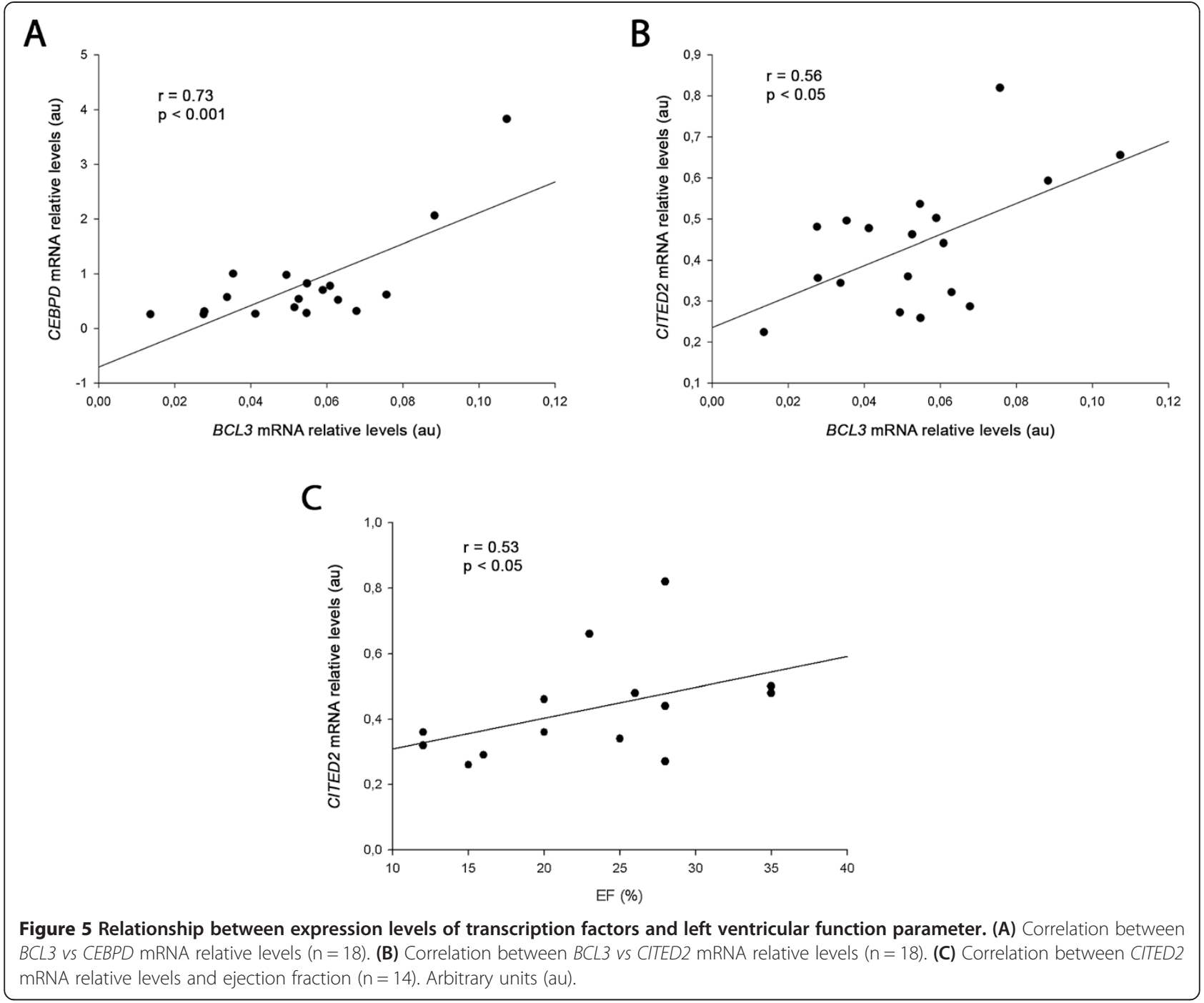

activation of prolonged-hypoxic response in ICM. Surprisingly, we did not observe changes in the expression of other hypoxia-inducible angiogenic genes, such as ANGPT1 or VEGF, which would reflect the defect in vascular growth in ICM patients. Moreover, GO functional analysis did not show terms related with angiogenesis indicating that angiogenic capacity is not affected by decreased expression of CITED2. These results are consistent with the well-characterized, defects in angiogenesis that is seen in ICM $[51,52]$. Evidence suggests that impaired capillary growth and the resulting metabolic imbalance are important contributors to the transition to HF. Furthermore, our data suggest that the downregulation of the CEBPD pathway suppresses angiogenesis through decreased expression of TGFBR [53]. On the other hand, decreased expression of CITED2 has been shown to trigger defects in cardiovascular development via the NodalPitx2c pathway in the mouse [54]. We did not observe changes in PITX2 expression between CNT and ICM, suggesting that this pathway is not compromised in ICM patients.

Finally, the correlation of $B C L 3$ with both $C E B P D$ and CITED2 suggests a possible new mechanism of cooperative regulation. Although a direct interaction between $B C L 3$ and $C E B P D$ has not been previously shown, both are involved in apoptosis [55]. Further studies are needed to shed light on their relation. The correlation between CITED2 mRNA levels and the EF clinical parameter demonstrate the relevance of CITED2 in heart function. Recent findings demonstrating that hypoxia reduces $\mathrm{EF}$ underscore the role of CITED2 in ICM [56].

In this study, we analyzed TFs mRNA levels. Additional levels of control, such as post-transcriptional modifications or cell compartmentalization, are critical for TF activity, and must be considered to understand their roles in ICM. For example, we have previously shown that disruption of the nuclear pore architecture, as well as changes in nucleocytoplasmic transporters at the mRNA and protein 


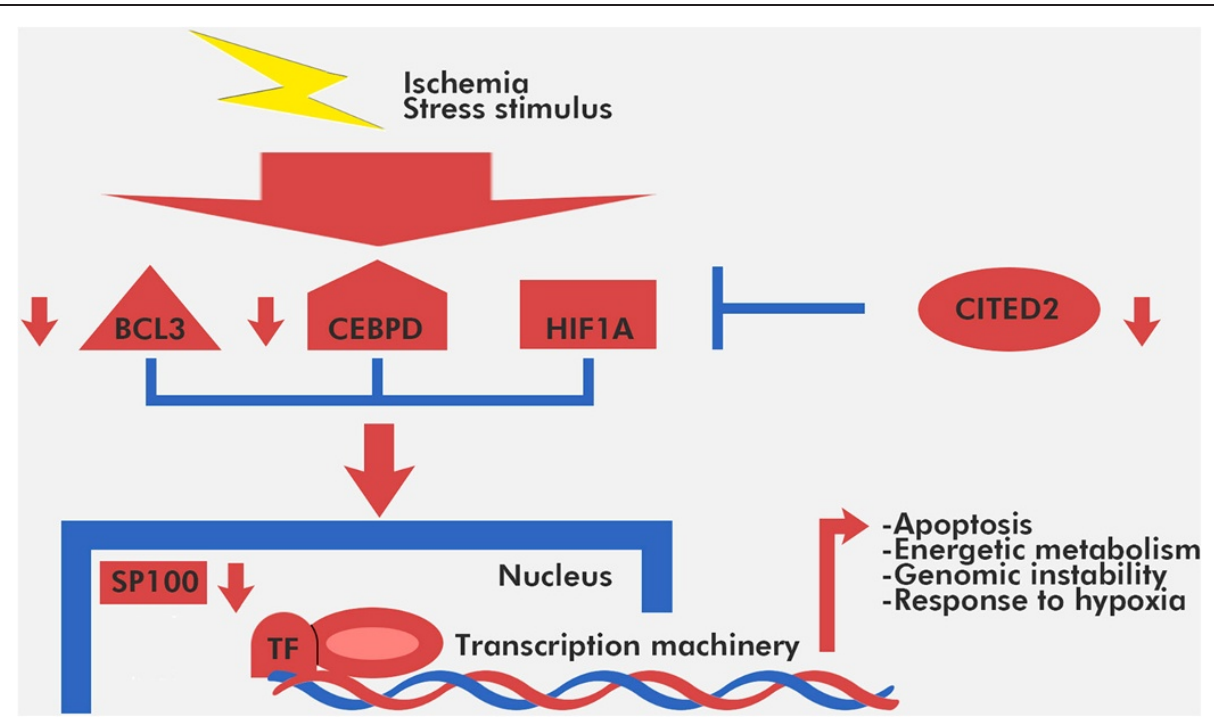

Figure 6 Hypothetical model of the transcriptional regulatory network in ICM patients. Expression of the TFs, CITED2, CEBPD and BCL3, and SP100 is downregulated under conditions of hypoxia and other stress stimuli, which in turn modifies the expression of their target gene networks. These changes ultimately result in alterations in cellular processes that are characteristic of ICM.

levels are characteristic of ICM patients [57,58]. Accordingly, alterations in the mRNA levels of TFs may contribute to ICM, but are not necessarily the only mechanism involved in the regulation of their downstream, responsive genes.

A common limitation of studies that use cardiac tissues from end-stage failing human hearts, including this study, is the high variability in disease etiology and in the treatment of the patients. Another issue is that this study was performed in heart tissue and not in isolated cardiomyocytes; however, we have analyzed these samples using confocal and electron microscopy. Images show that cardiomyocytes are the majority of the cell population. It is also noteworthy that this study was conducted using a large number of human heart samples, including samples from CNT hearts. This makes our data valuable for studies in ICM patients.

\section{Conclusions}

The newly described differences in the expression of SP100, BCL3, CITED2, and CEBPD between ICM and CNT samples contributes to our understanding of alterations in crucial biological processes, including apoptosis, stress, energetic metabolism and hypoxic response, in ICM patients. The relationship between CITED2 expression and EF emphasizes the relevance of this factor in ICM. Our results show new evidence for a dysregulated transcriptional network in ICM patients. Moreover, our findings provide valuable resources for further studies of the molecular mechanism in heart ischemic response and potential novel biomarkers of ICM.

\section{Additional files}

Additional file 1: Table S1. Quality of sample mapping.

Additional file 2: Figure S1. Correlation of read counts between replicates. Correlation heat map for samples used by RNA-Seq analysis $(n=19)$. Correlation coefficient scores showed that Pearson correlation between samples was 0.85 . There were not clear outliers relative to the other samples.

Additional file 3: Table S2. Completed list of TF identified in ChEA database using the transcriptional signature obtained by RNA-Seq.

Additional file 4: Table S3. TF target genes expression levels calculated by RNA-Seq.

Additional file 5: Table S4. Additional GO associated to the downstream target genes of CEBPD, BCL3 and HIF1A.

\section{Abbreviations}

RNA-Seq: RNA-Sequencing; LV: Left ventricular; EF: Ejection fraction; ICM: Ischemic cardiomyopathy; HF: Heart failure; CNT: Control; TF: Transcription factor; NT: Nucleotide; RNA: Ribonucleic acid; RIN: RNA integrity number; CDNA: Complementary deoxyribonucleic acid; qRT-PCR: Quantitative Real-Time PCR; PCA: Principal component analysis; SP100: SP100 nuclear antigen; CITED2: Cbp/p300-interacting transactivator 2; CEBPD: CCAAT/enhancer binding protein delta; BCL3: B-cell CLL/lymphoma 3; GO: Gene ontology.

\section{Competing interests}

The authors declare that they have no competing interests.

\section{Authors' contributions}

$\mathrm{IH}$ carried out all the experiments and data analysis, conceived of present study, and drafted and wrote the manuscript. ERL, AO, ET, and MMMN collected all samples, contributed to the experiments and were involved in manuscript revision. JCT contributed to RNA-Seq bioinformatic analysis. LMD, $L A, F L, I S L, J R G J$, and AS contributed to the discussion of the findings. $I H$ and MP participated in data analysis and in the manuscript revision. MR conceived and designed the study, participated in data analysis and the manuscript revision. All authors read and approved the final manuscript. 


\section{Acknowledgments}

We thank the Transplant Coordination Unit (Hospital Universitario La Fe, Valencia, Spain) for their help in obtaining the heart tissue samples and Andrea Montesa Malenza for his designer support. This research was supported by grants from the National Institute of Health "Fondo de Investigaciones Sanitarias del Instituto de Salud Carlos III" [Retics RD12/0042/ 0003 and RD06/003/1001, and FIS Projects PI10/00275 and PI13/00100], and co-financed by The European Regional Development Fund (FEDER).

\section{Author details}

${ }^{1}$ Cardiocirculatory Unit, Health Research Institute of La Fe University Hospital, Avd de Fernando Abril Martorell, 106, 46026 Valencia, Spain. ${ }^{2}$ Genomic Systems, Paterna, Valencia, Spain. ${ }^{3}$ Heart Failure and Transplantation Unit, Cardiology Department, La Fe University Hospital, Valencia, Spain. ${ }^{4}$ Cellular and Molecular Cardiology Unit, Department of Cardiology and Institute of Biomedical Research, University Clinical Hospital, Santiago Compostela, Spain.

Received: 31 May 2014 Accepted: 10 March 2015

Published online: 29 March 2015

\section{References}

1. Yellon DM, Hausenloy DJ. Myocardial reperfusion injury. N Engl J Med. 2007;357:1121-35.

2. Hasenfuss G, Pieske B. Calcium cycling in congestive heart failure. J Mol Cell Cardiol. 2002;34:951-69.

3. Cicconi S, Ventura N, Pastore D, Bonini P, Di Nardo P, Lauro R, et al. Characterization of apoptosis signal transduction pathways in $\mathrm{HL}-5$ cardiomyocytes exposed to ischemia/reperfusion oxidative stress model. J Cell Physiol. 2003;195:27-37.

4. Hoshijima M, Chien KR. Mixed signals in heart failure: cancer rules. J Clin Invest. 2002;109:849-55.

5. Rajabi M, Kassiotis C, Razeghi P, Taegtmeyer H. Return to the fetal gene program protects the stressed heart: a strong hypothesis. Heart Fail Rev. 2007;12:331-43.

6. Kittleson MM, Minhas KM, Irizarry RA, Ye SQ, Edness G, Breton E, et al. Gene expression analysis of ischemic and nonischemic cardiomyopathy: shared and distinct genes in the development of heart failure. Physiol Genomics. 2005;21:299-07.

7. Ahmad F, Seidman JG, Seidman CE. The genetic basis for cardiac remodeling. Annu Rev Genomics Hum Genet. 2005;6:185-216.

8. Cortés R, Rivera M, Roselló-Lletí E, Martínez-Dolz L, Almenar L, Azorín I, et al. Differences in MEF2 and NFAT transcriptional pathways according to human heart failure aetiology. PLoS One. 2012;7:e30915.

9. Pashmforoush M, Lu JT, Chen H, Amand TS, Kondo R, Pradervand S, et al. Nkx2-5 pathways and congenital heart disease; loss of ventricular myocyte lineage specification leads to progressive cardiomyopathy and complete heart block. Cell. 2004;117:373-86.

10. Li C, Browder W, Kao RL. Early activation of transcription factor NF-kappaB during ischemia in perfused rat heart. Am J Physiol. 1999;276:H543-52.

11. Haghikia A, Stapel B, Hoch M, Hilfiker-Kleiner D. STAT3 and cardiac remodeling. Heart Fail Rev. 2011;16:35-47.

12. Windak R, Müller J, Felley A, Akhmedov A, Wagner EF, Pedrazzini T, et al. The AP-1 Transcription Factor c-Jun Prevents Stress-Imposed Maladaptive Remodeling of the Heart. PLoS One. 2013;8:e73294.

13. Chen XL, Lei YH, Liu CF, Yang QF, Zuo PY, Liu CY, et al. Angiogenesis inhibitor bevacizumab increases the risk of ischemic heart disease associated with chemotherapy: a meta-analysis. PLoS One. 2013;8:e66721.

14. Knoops L, Haas R, de Kemp S, Majoor D, Broeks A, Eldering E, et al. In vivo p53 response and immune reaction underlie highly effective low-dose radiotherapy in follicular lymphoma. Blood. 2007;110:1116-12.

15. Chen HB, Wang L, Jiang JF. Re-analysis of expression profiles for revealing new potential candidate genes of heart failure. Eur Rev Med Pharmacol Sci. 2013;17:903-11.

16. Nalpas NC, Park SD, Magee DA, Taraktsoglou M, Browne JA, Conlon KM, et al. Whole-transcriptome, high-throughput RNA sequence analysis of the bovine macrophage response to Mycobacterium bovis infection in vitro. BMC Genomics. 2013;14:230.

17. Macrae DJ. The Council for International Organizations and Medical Sciences (CIOMS) guidelines on ethics of clinical trials. Proc Am Thorac Soc. 2007:4:176-8
18. McMurray JJ, Adamopoulos S, Anker SD, Auricchio A, Böhm M, Dickstein K, et al. ESC Guidelines for the diagnosis and treatment of acute and chronic heart failure 2012: The Task Force for the Diagnosis and Treatment of Acute and Chronic Heart Failure 2012 of the European Society of Cardiology. Developed in collaboration with the Heart Failure Association (HFA) of the ESC. Eur Heart J. 2012;33:1787-847.

19. Li H, Handsaker B, Wysoker A, Fennell T, Ruan J, Homer N, et al. The Sequence Alignment/Map format and SAM tools. Bioinformatics. 2009;25:2078-9.

20. Trapnell C, Williams BA, Pertea G, Mortazavi A, Kwan G, van Baren MJ, et al. Transcript assembly and quantification by RNA-Seq reveals unannotated transcripts and isoform switching during cell differentiation. Nat Biotechnol. 2010;28:511-5

21. Robinson MD, McCarthy DJ, Smyth GK. edgeR: a Bioconductor package for differential expression analysis of digital gene expression data. Bioinformatics. 2010;26:139-40.

22. Lachmann A, Xu H, Krishnan J, Berger SI, Mazloom AR, Ma'ayan A. ChEA: transcription factor regulation inferred from integrating genome-wide ChIP-X experiments. Bioinformatics. 2010;26:2438-44.

23. Livak KJ, Schmittgen TD. Analysis of relative gene expression data using real-time quantitative PCR and the 2(-Delta Delta C (T)) Method. Methods. 2001;25:402-8.

24. da Huang W, Sherman BT, Lempicki RA. Systematic and integrative analysis of large gene lists using DAVID bioinformatics resources. Nat Protoc. 2009:4:44-57.

25. Eom GH, Nam YS, Oh JG, Choe N, Min HK, Yoo EK, et al. Regulation of acetylation of histone deacetylase 2 by p300/CBP-associated factor/histone deacetylase 5 in the development of cardiac hypertrophy. Circ Res. 2014;114:1133-43.

26. Bakker WJ, Harris IS, Mak TW. FOXO3a is activated in response to hypoxic stress and inhibits HIF1-induced apoptosis via regulation of CITED2. Mol Cell. 2007;28:941-53.

27. Yang J, Williams RS, Kelly DP. Bcl3 interacts cooperatively with peroxisome proliferator-activated receptor gamma (PPARgamma) coactivator 1alpha to coactivate nuclear receptors estrogen-related receptor alpha and PPARalpha. Mol Cell Biol. 2009;29:4091-102.

28. Seeler JS, Marchio A, Losson R, Desterro JMP, Hay RT, Chambon P, et al. Common properties of nuclear body protein SP100 and TIF1alpha chromatin factor: role of SUMO modification. Mol Cell Biol. 2001;21:3314-24.

29. Shin DH, Li SH, Chun YS, Huang LE, Kim MS, Park JW. CITED2 mediates the paradoxical responses of HIF-1alpha to proteasome inhibition. Oncogene. 2008;27:1939-44.

30. Benita Y, Kikuchi $H$, Smith AD, Zhang MQ, Chung DC, Xavier RJ. An integrative genomics approach identifies Hypoxia Inducible Factor-1 (HIF-1)-target genes that form the core response to hypoxia. Nucleic Acids Res. 2009;37:4587-602.

31. Schlesinger J, Schueler M, Grunert M, Fischer JJ, Zhang Q, Krueger T, et al. The cardiac transcription network modulated by Gata4, Mef2a, Nkx2.5, Srf, histone modifications, and microRNAs. PLoS Genet. 2011;7:e1001313.

32. Yang J, Moravec CS, Sussman MA, DiPaola NR, Fu D, Hawthorn L, et al. Decreased SLIM1 expression and increased gelsolin expression in failing human hearts measured by high-density oligonucleotide arrays. Circulation. 2000;102:3046-52.

33. Wei JQ, Shehadeh LA, Mitrani JM, Pessanha M, Slepak TI, Webster KA, et al. Quantitative control of adaptive cardiac hypertrophy by acetyltransferase p300. Circulation. 2008:118:934-46.

34. Yilmaz A, Menevse S, Erkan AF, Ergun MA, Ilhan MN, Cengel A, et al. The relationship of the ESR1 gene polymorphisms with the presence of coronary artery disease determined by coronary angiography. Genet Test. 2007;11:367-71.

35. Loh YH, Wu Q, Chew JL, Vega VB, Zhang W, Chen X, et al. The Oct4 and Nanog transcription network regulates pluripotency in mouse embryonic stem cells. Nat Genet. 2006;38:431-40.

36. Seeler JS, Marchio A, Sitterlin D, Transy C, Dejean A. Interaction of SP100 with HP1 proteins: a link between the promyelocytic leukemia-associated nuclear bodies and the chromatin compartment. Proc Natl Acad Sci U S A 1998;95:7316-21

37. Negorev DG, Vladimirova OV, Kossenkov AV, Nikonova EV, Demarest RM, Capobianco AJ, et al. Sp100 as a potent tumor suppressor: accelerated senescence and rapid malignant transformation of human fibroblasts through modulation of an embryonic stem cell program. Cancer Res. 2010;70:9991-01.

38. Haider N, Narula N, Narula J. Apoptosis in heart failure represents programmed cell survival, not death, of cardiomyocytes and likelihood of reverse remodeling. J Card Fail. 2002;8:S512-7. 
39. Hoshino A, Mita Y, Okawa Y, Ariyoshi M, Iwai-Kanai E, Ueyama T, et al. Cytosolic p53 inhibits Parkin-mediated mitophagy and promotes mitochondrial dysfunction in the mouse heart. Nat Commun. 2013;4:2308.

40. Huang Z, Rivas B, Agoulnik Al. Insulin-like 3 signaling is important for testicular descent but dispensable for spermatogenesis and germ cell survival in adult mice. Biol Reprod. 2012:87:143.

41. Novellasdemunt L, Tato I, Navarro-Sabate A, Ruiz-Meana M, Méndez-Lucas A, Perales JC, et al. Akt-dependent activation of the heart 6-phosphofructo2-kinase/fructose-2,6-bisphosphatase (PFKFB2) isoenzyme by amino acids. J Biol Chem. 2013;288:10640-51.

42. Zhang Q, Didonato JA, Karin M, McKeithan TW. BCL3 encodes a nuclear protein which can alter the subcellular location of NF-kappaB proteins. Mol Cell Biol. 1994:14:3915-26.

43. Bours V, Franzoso G, Azarenko V, Park S, Kanno T, Brown K, et al. The oncoprotein $\mathrm{BCl}-3$ directly transactivates through kappa B motifs via association with DNA-binding p50B homodimers. Cell. 1993;72:729-39.

44. Wong SC, Fukuchi M, Melnyk P, Rodger I, Giaid A. Induction of cyclooxygenase-2 and activation of nuclear factor-kB in myocardium of patients with congestive heart failure. Circulation. 1998;98:100-3.

45. Grabellus F, Levkau B, Sokoll A, Welp H, Schmid C, Deng MC, et al Reversible activation of nuclear factor-kB in human end-stage heart failure after left ventricular mechanical support. Cardiovasc Res. 2002:53:124-30.

46. Yoon H, Lim JH, Cho CH, Huang LE, Park JW. CITED2 controls the hypoxic signaling by snatching p300 from the two distinct activation domains of HIF-1a. Biochim Biophys Acta. 1813;2011:2008-16.

47. Villar D, Ortiz-Barahona A, Gómez-Maldonado L, Pescador N, Sánchez-Cabo $\mathrm{F}$, Hackl H, et al. Cooperativity of stress-responsive transcription factors in core hypoxia-inducible factor binding regions. PLoS One. 2012;7:e45708.

48. Semenza GL. Regulation of oxygen homeostasis by hypoxia-inducible factor 1. Physiology (Bethesda). 2009;24:97-106.

49. Eckle T, Köhler D, Lehmann R, El Kasmi KC, Eltzschig HK. Hypoxia-inducible factor-1 is central to cardioprotection: a new paradigm for ischemic preconditioning. Circulation. 2008;118:166-75.

50. Lei L, Mason S, Liu D, Huang Y, Marks C, Hickey R, et al. Hypoxia-inducible factor-dependent degeneration, failure, and malignant transformation of the heart in the absence of the von Hippel-Lindau protein. Mol Cell Biol. 2008:28:3790-803.

51. Nessa A, Latif SA, Siddiqui NI, Hussain MA, Bhuiyan MR, Hossain MA, et al. Angiogenesis-a novel therapeutic approach for ischemic heart disease Mymensingh Med J. 2009;18:264-72.

52. Roura S, Planas F, Prat-Vidal C, Leta R, Soler-Botija C, Carreras F, et al. Idiopathic dilated cardiomyopathy exhibits defective vascularization and vessel formation. Eur J Heart Fail. 2007;9:995-02.

53. Oh SP, Seki T, Goss KA, Imamura T, Yi Y, Donahoe PK, et al. Activin receptorlike kinase 1 modulates transforming growth factor-beta 1 signaling in the regulation of angiogenesis. Proc Natl Acad Sci U S A. 2000;97:2626-31.

54. Bamforth SD, Bragança J, Farthing CR, Schneider JE, Broadbent C, Michell $A C$, et al. Cited 2 controls left-right patterning and heart development through a Nodal-Pitx2C pathway. Nat Genet. 2004;36:1189-96.

55. Bauer A, Villunger A, Labi V, Fischer SF, Strasser A, Wagner $H$, et al. The NF-kappaB regulator $\mathrm{BCl}-3$ and the $\mathrm{BH} 3$-only proteins Bim and Puma control the death of activated T cells. Proc Natl Acad Sci U S A. 2006;103:10979-84.

56. Silverman NA, Kohler J, Levitsky S, Pavel DG, Fang RB, Feinberg H. Chronic hypoxemia depresses global ventricular function and predisposes to the depletion of high-energy phosphates during cardioplegic arrest: implications for surgical repair of cyanotic congenital heart defects. Ann Thorac Surg. 1984;37:304-8.

57. Molina-Navarro MM, Roselló-Lletí E, Tarazón E, Ortega A, Sánchez-Izquierdo $D$, Lago $F$, et al. Heart failure entails significant changes in human nucleocytoplasmic transport gene expression. Int J Cardiol. 2013;168:2837-43.

58. Tarazón E, Rivera M, Roselló-Lletí E, Molina-Navarro MM, Sánchez-Lázaro IJ, España F, et al. Heart failure induces significant changes in nuclear pore complex of human cardiomyocytes. PLoS One. 2012;7:e48957.

\section{Submit your next manuscript to BioMed Central and take full advantage of:}

- Convenient online submission

- Thorough peer review

- No space constraints or color figure charges

- Immediate publication on acceptance

- Inclusion in PubMed, CAS, Scopus and Google Scholar

- Research which is freely available for redistribution

Submit your manuscript at www.biomedcentral.com/submit 